\title{
ON THE $L^{1}$-CONVERGENCE OF FOURIER TRANSFORMS
}

\author{
DĀNG VŨ GIANG and FERENC MÓRICZ
}

(Received 15 December 1994)

Communicated by A. H. Dooley

\begin{abstract}
We study cosine and sine Fourier transforms defined by $F(t):=(2 / \pi) \int_{0}^{\infty} f(x) \cos t x d x$ and $\tilde{F}(t):=$ $(2 / \pi) \int_{0}^{\infty} f(x) \sin t x d x$, where $f$ is $L^{1}$-integrable over $[0, \infty)$. We also assume that $F$ and $\tilde{F}$ are locally absolutely continuous over $[0, \infty)$. In particular, this is the case if both $f(x)$ and $x f(x)$ are $L^{1}$ integrable over $[0, \infty)$. Motivated by the inversion formulas, we consider the partial integrals $s_{v}(f, x):=$ $\int_{0}^{v} F(t) \cos x t d t$ and $\tilde{s}_{v}(f, x):=\int_{0}^{\nu} \tilde{F}(t) \sin x t d t$, the modified partial integrals $u_{v}(f, x):=s_{v}(f, x)-$ $F(v)(\sin v x) / x$ and $\tilde{u}_{v}(f, x):=\tilde{s}_{v}(f, x)+\tilde{F}(v)(\cos v x) / x$, where $v>0$. We give necessary and sufficient conditions for the $L^{1}[0, \infty)$-convergence of $u_{v}(f)$ and $\tilde{u}_{v}(f)$ as well as for the $L^{1}[0, X]$ convergence of $s_{\nu}(f)$ and $\tilde{s}_{\nu}(f)$ to $f$ as $v \rightarrow \infty$, where $0<X<\infty$ is fixed. On the other hand, in certain cases we conclude that $s_{v}(f)$ and $\tilde{s}_{v}(f)$ cannot belong to $L^{1}[0, \infty)$. Consequently, it makes no sense to speak of their $L^{1}[0, \infty)$-convergence as $v \rightarrow \infty$.

As an intermediate tool, we use the Cesàro means of Fourier transforms. Then we prove Tauberian type results and apply Sidon type inequalities in order to obtain Tauberian conditions of Hardy-Karamata kind.

We extend these results to the complex Fourier transform defined by $G(t):=1 /(2 \pi) \int_{-\infty}^{\infty} g(t) e^{-i x t} d t$, where $g$ is $L^{1}$-integrable over $(-\infty, \infty)$.

1991 Mathematics subject classification (Amer. Math. Soc.): primary 42A38; secondary 26A46.

Keywords and phrases: cosine and sine Fourier transforms, complex Fourier transform, absolute continuity, $L^{1}$-convergence, inversion formula, partial integral, Cesàro mean, modified partial integral, Tauberian condition of Hardy-Karamata kind, Sidon type inequalities.
\end{abstract}

\section{Notation and basic assumptions}

We consider a complex-valued function $f$ that is Lebesgue integrable over $[0, \infty)$, in symbols : $f \in L^{1}[0, \infty)$. The cosine and sine Fourier transforms of $f$ are defined

This research was partially supported by the Hungarian National Foundation for Scientific Research under Grant \# T 016393.

(C) 1996 Australian Mathematical Society $0263-6115 / 96 \$ A 2.00+0.00$ 
respectively by

$$
\begin{aligned}
& F(t):=\frac{2}{\pi} \int_{0}^{\infty} f(x) \cos t x d x \\
& \tilde{F}(t):=\frac{2}{\pi} \int_{0}^{\infty} f(x) \sin t x d x \quad(t \geq 0) .
\end{aligned}
$$

As is well-known (see for example [5, pp. 13 and 16-17]), the inversion formulas

$$
\begin{aligned}
f(x) & =\lim _{\nu \rightarrow \infty} \int_{0}^{\nu} F(t) \cos x t d t \\
& =\lim _{\nu \rightarrow \infty} \int_{0}^{\nu} \tilde{F}(t) \sin x t d t \quad(x>0)
\end{aligned}
$$

hold true under various conditions. The aim of this paper is to recover $f$ from $F$ or $\tilde{F}$ by means of $L^{1}$-convergence.

To this effect, we consider the partial integrals of (1.3) defined by

$$
\begin{aligned}
& s_{v}(f, x):=\int_{0}^{\nu} F(t) \cos x t d t \\
& \tilde{s}_{v}(f, x):=\int_{0}^{\nu} \tilde{F}(t) \sin x t d t
\end{aligned}
$$

the Cesàro means, or $(C, 1)$ means of $(1.3)$ defined by

$$
\begin{aligned}
& \sigma_{\nu}(f, x):=\int_{0}^{\nu}\left(1-\frac{t}{v}\right) F(t) \cos x t d t \\
& \tilde{\sigma}_{\nu}(f, x):=\int_{0}^{\nu}\left(1-\frac{t}{v}\right) \tilde{F}(t) \sin x t d t
\end{aligned}
$$

(see for example [5, p. 26]), where $v>0$ and $x \geq 0$ are arbitrary real numbers.

The usage of $\sigma_{v}(f)$ and $\tilde{\sigma}_{v}(f)$ is analogous to the summability of series by the method of the first arithmetic mean, which is too well-known to need much discussion here. (See for example [6, Vol. 1, Ch. 3].)

From now on, we also assume that $F$ and $\tilde{F}$ are locally absolutely continuous (in abbreviation: LAC) over $[0, \infty)$. In particular, this is the case if both $f(x)$ and $x f(x)$ belong to $L^{1}[0, \infty)$. In fact, then the cosine Fourier transform $F$ is differentiable and

$$
F^{\prime}(t)=-\tilde{F}_{1}(t) \quad(t \geq 0),
$$

where $\tilde{F}_{1}$ is the sine Fourier transform of $f_{1}(x):=x f(x)$.

The last statement is a common place. But for the reader's convenience, we prove it briefly. The function

$$
f(x) \frac{\cos (t+h) x-\cos t x}{h}=f(x) \cos t x \frac{\cos h x-1}{h}-x f(x) \sin t x \frac{\sin h x}{h x}
$$


is majorized in absolute value by the integrable function $|x f(x)|$ independent of $h$, and tends to the limit $-x f(x) \sin t x$ as $h \rightarrow 0$. By the Lebesgue dominated convergence theorem,

$$
\lim _{h \rightarrow 0} \frac{F(t+h)-F(t)}{h}=-\tilde{F}_{1}(t) \quad(t \geq 0),
$$

which is (1.4). (In case $t=0$, we consider $h>0$ only.)

Making use of definition (1.2) and Fubini's theorem results in the following

$$
\begin{aligned}
\int_{0}^{t} F^{\prime}(u) d u & =-\int_{0}^{t} \int_{0}^{\infty} x f(x) \sin u x d x d u=-\int_{0}^{\infty} x f(x) \int_{0}^{t} \sin u x d u d x \\
& =\int_{0}^{\infty} f(x)(\cos t x-1) d x=F(t)-F(0) \quad(t \geq 0) .
\end{aligned}
$$

This shows that $F$ is indeed LAC over $[0, \infty)$.

The statement that $\tilde{F}$ is also LAC over $[0, \infty)$ can be proved analogously. In particular, we have

$$
\tilde{F}^{\prime}(t)=F_{1}(t) \quad(t \geq 0),
$$

where $F_{1}$ is the cosine Fourier transform of $f_{1}(x)$.

Next, we introduce the auxiliary expressions

$$
\begin{aligned}
& \tau_{v}(f, \lambda, x):=\frac{\lambda}{\lambda-1} \int_{v}^{\lambda v}\left(1-\frac{t}{\lambda \nu}\right) F^{\prime}(t) \frac{\sin x t}{x} d t, \\
& \tilde{\tau}_{v}(f, \lambda, x):=-\frac{\lambda}{\lambda-1} \int_{v}^{\lambda v}\left(1-\frac{t}{\lambda v}\right) \tilde{F}^{\prime}(t) \frac{\cos x t}{x} d t,
\end{aligned}
$$

where $\nu>0, \lambda>1$ and $x>0$ are arbitrary real numbers. They will play a decisive role in our results on $L^{1}$-convergence.

By integration by parts, we obtain

$$
s_{v}(f, x)=F(v) \frac{\sin v x}{x}-\int_{0}^{v} F^{\prime}(t) \frac{\sin x t}{x} d t .
$$

This motivates the introduction of the modified partial integral defined by

$$
u_{v}(f, x):=s_{v}(f, x)-F(v) \frac{\sin v x}{x}=-\int_{0}^{v} F^{\prime}(t) \frac{\sin x t}{x} d t,
$$

and analogously,

$$
\tilde{u}_{v}(f, x):=\tilde{s}_{v}(f, x)+\tilde{F}(v) \frac{\cos v x}{x}=\int_{0}^{v} \tilde{F}^{\prime}(t) \frac{\cos x t}{x} d t .
$$




\section{Main results}

We characterize the $L^{1}[0, \infty)$-convergence of $u_{v}(f)$ and $\tilde{u}_{v}(f)$ to $f$ as $v \rightarrow \infty$.

THEOREM 1. If $f \in L^{1}[0, \infty)$ and $F$ is LAC over $[0, \infty)$, then

$$
\lim _{v \rightarrow \infty} \int_{0}^{\infty}\left|u_{v}(f, x)-f(x)\right| d x=0
$$

if and only if

$$
\lim _{\lambda \downarrow 1} \limsup _{\nu \rightarrow \infty} \int_{0}^{\infty}\left|\tau_{\nu}(f, \lambda, x)\right| d x=0
$$

THEOREM 2. If $f \in L^{1}[0, \infty)$ and $\tilde{F}$ is LAC over $[0, \infty)$, then

$$
\lim _{\nu \rightarrow \infty} \int_{1 / \nu}^{\infty}\left|\tilde{u}_{\nu}(f, x)-f(x)\right| d x=0
$$

if and only if

$$
\lim _{\lambda \downarrow 1} \limsup _{v \rightarrow \infty} \int_{1 / \nu}^{\infty}\left|\tilde{\tau}_{v}(f, \lambda, x)\right| d x=0
$$

It is an easy consequence that, under the conditions of Theorems 1 and 2, we cannot expect the $L^{1}[0, \infty)$-convergence of $s_{v}(f)$ and $\tilde{s}_{v}(f)$ as $v \rightarrow \infty$, respectively.

COROLlaRY 1. If $f \in L^{1}[0, \infty), F$ is LAC over $[0, \infty)$, and condition (2.2) is satisfied, then $s_{v}(f) \notin L^{1}[0, \infty)$ for each $v$ such that $F(v) \neq 0$.

COROLlary 2. If $f \in L^{1}[0, \infty), \tilde{F}$ is LAC over $[0, \infty)$, and condition (2.4) is satisfied, then $\tilde{s}_{v}(f) \notin L^{1}[0, \infty)$ for each $v$ such that $\tilde{F}(v) \neq 0$.

On the other hand, we are able to characterize the $L^{1}$-convergence of $s_{\nu}(f)$ and $\tilde{s}_{v}(f)$ to $f$ over any finite interval $[0, X]$ as $\nu \rightarrow \infty$, where $0<X<\infty$ is arbitrary.

COROLlaRY 3. If $f \in L^{1}[0, \infty), F$ is LAC over $[0, \infty)$, and condition (2.2) is satisfied, then for any $0<X<\infty$

$$
\lim _{\nu \rightarrow \infty} \int_{0}^{x}\left|s_{v}(f, x)-f(x)\right| d x=0 \text { if and only if } \lim _{\nu \rightarrow \infty} F(v) \ln v=0 .
$$


COROllary 4. If $f \in L^{1}[0, \infty), \tilde{F}$ is LAC over $[0, \infty)$, and condition (2.4) is satisfied, then for any $0<X<\infty$

$$
\lim _{\nu \rightarrow \infty} \int_{0}^{X}\left|\tilde{s}_{\nu}(f, x)-f(x)\right| d x=0 \text { if and only if } \lim _{\nu \rightarrow \infty} \tilde{F}(\nu) \ln \nu=0 .
$$

The following simple example shows that there exists a function $f \in L^{1}[0, \infty)$ such that its cosine Fourier transform $F$ is absolutely continuous over $[0, \infty), F(v) \ln v \rightarrow$ 0 as $v \rightarrow \infty$, but $s_{v}(f) \notin L^{1}[0, \infty)$ for all $v>0$. Consequently, it makes no sense to speak of the $L^{1}[0, \infty)$-convergence of $s_{v}(f)$ as $v \rightarrow \infty$.

EXAMPLE. Let $F(t):=2 / \pi(t+1)$ for $t \geq 0$,

$$
f(x):=\lim _{\nu \rightarrow \infty} \int_{0}^{v} F(t) \cos x t d t \quad(x>0) .
$$

It is plain that

$$
\int_{0}^{\infty}\left(\operatorname{ess} \sup _{u \leq t \leq 2 u}\left|F^{\prime}(t)\right|\right) d u<\infty
$$

By [3, Theorem 1], it follows that $f \in L^{1}[0, \infty)$. Keeping the inversion formula (1.3) in mind, by Corollary 5 below, we conclude (2.1). By (1.5), the difference $s_{\nu}(f)-u_{\nu}(f)$, and a fortiori $s_{\nu}(f)$, does not belong to $L^{1}[0, \infty)$ for any $\nu>0$.

We note that an easy computation gives that

$$
\begin{gathered}
f(x)=\frac{2}{\pi} \int_{0}^{\infty} \frac{\sin x t}{(1+t)^{2} x} d t, \\
u_{v}(f, x)-f(x)=-\frac{2}{\pi} \int_{v}^{\infty} \frac{\sin x t}{(1+t)^{2} x} d t, \\
s_{\nu}(f, x)-f(x)=-\frac{2}{\pi} \int_{\nu}^{\infty} \frac{\sin x t}{(1+t)^{2} x} d t+\frac{2 \sin v x}{\pi(1+v) x},
\end{gathered}
$$

whence the above statements can be checked directly, too.

PROBLeM 1. It is an open problem how to deduce sufficient or/and necessary conditions for the $L^{1}[0, \infty)$-convergence of $s_{v}(f)$ and $\tilde{s}_{v}(f)$ to $f$ as $v \rightarrow \infty$ ? Since the differences $u_{v}(f)-s_{v}(f)$ and $\tilde{u}_{v}(f)-\tilde{s}_{v}(f)$ do not belong to $L^{1}[0, \infty)$ (cf. (1.5) and (1.6)), one should avoid the usage of $u_{v}(f)$ and $\tilde{u}_{v}(f)$ during the solution of this problem.

According to Theorems 1,2 and Corollaries 3,4 , the question of $L^{1}$-convergence of $u_{v}(f), \tilde{u}_{v}(f), s_{v}(f)$, and $\tilde{s}_{v}(f)$ reduces to finding sufficient conditions for the fulfillment of (2.2) and (2.4), respectively. 
THEOREM 3. If $f \in L^{1}[0, \infty), F$ is LAC over $[0, \infty)$, and for some $p>1$ we have

$$
\lim _{\lambda \downarrow 1} \limsup _{\nu \rightarrow \infty} \int_{\nu}^{\lambda \nu} t^{p-1}\left|F^{\prime}(t)\right|^{p} d t<\infty
$$

then condition (2.2) is satisfied.

COROllary 5. If $f \in L^{1}[0, \infty), F$ is LAC over $[0, \infty)$, and

$$
t F^{\prime}(t)=\mathscr{O}(1) \text { as } t \rightarrow \infty,
$$

then condition (2.2) is satisfied.

THEOREM 4. If $f \in L^{1}[0, \infty), \tilde{F}$ is $\mathrm{LAC}$ over $[0, \infty)$, and for some $p>1$ we have

$$
\lim _{\lambda \downarrow 1} \limsup _{v \rightarrow \infty} \int_{v}^{\lambda v} t^{p-1}\left|\tilde{F}^{\prime}(t)\right|^{p} d t<\infty
$$

then condition (2.4) is satisfied.

COROllaRY 6. If $f \in L^{1}[0, \infty), \tilde{F}$ is LAC over $[0, \infty)$, and

$$
t \tilde{F}^{\prime}(t)=\mathscr{O}(1) \text { as } t \rightarrow \infty,
$$

then condition (2.4) is satisfied.

Also, conditions (2.7), or in particular (2.8), are sufficient for the fulfillment of both (2.1) and (2.5). Likewise, conditions (2.9), or in particular (2.10), are sufficient for the fulfillment of both (2.3) and (2.6).

A few comments are appropriate here.

(i) Theorems 3 and 4 are stronger when $p$ is closer to 1. By Hölder's inequality, if $0<p_{1}<p_{2}<\infty$, then

$$
\left(\int_{\nu}^{\lambda \nu} t^{p_{1}-1}\left|F^{\prime}(t)\right|^{p_{1}} d t\right)^{1 / p_{1}} \leq(\ln \lambda)^{\left(1 / p_{1}\right)-\left(1 / p_{2}\right)}\left(\int_{\nu}^{\lambda \nu} t^{p_{2}-1}\left|F^{\prime}(t)\right|^{p_{2}} d t\right)^{1 / p_{2}} .
$$

Thus, without loss of generality we may assume that for some $1<p \leq 2$ we actually have

$$
\lim _{i \downarrow 1} \limsup _{v \rightarrow \infty} \int_{v}^{\lambda v} t^{p-1}\left|F^{\prime}(t)\right|^{p} d t=0
$$


A similar observation pertains to condition (2.9). That is, we may assume that for some $1<p \leq 2$ we actually have

$$
\lim _{\lambda \downarrow 1} \limsup _{v \rightarrow \infty} \int_{v}^{\lambda v} t^{p-1}\left|\tilde{F}^{\prime}(t)\right|^{p} d t=0
$$

(ii) The counterparts of conditions $\left(2.7^{\prime}\right)$ and $\left(2.9^{\prime}\right)$ are well-known in the theory of Fourier series and called Tauberian conditions of Hardy-Karamata kind (see, for example $[1,2,4])$.

(iii) In the special case $p=1$, condition $\left(2.7^{\prime}\right)$ is of the form

$$
\lim _{\lambda \downarrow 1} \limsup _{v \rightarrow \infty} \int_{v}^{\lambda v}\left|F^{\prime}(t)\right| d t=0
$$

PROBLEM 2. We guess that condition (2.11) is not enough to ensure $L^{1}$-convergence. However, we are unable to construct a function $f \in L^{1}[0, \infty)$ such that its cosine Fourier transform $F$ is LAC over $[0, \infty)$, condition (2.11) is satisfied, and relation (2.1) is not satisfied.

We claim an analogous conjecture in connection with condition $\left(2.9^{\prime}\right)$.

\section{Auxiliary results}

We present four lemmas.

LeMmA 1. (See [5, p. 11].) If $f \in L^{1}[0, \infty)$, then $F(t)$ and $\tilde{F}(t)$ are continuous over $[0, \infty)$ and

$$
\lim _{t \rightarrow \infty} F(t)=\lim _{t \rightarrow \infty} \tilde{F}(t)=0
$$

LEMMA 2. (See [5, p. 35].) If $f \in L^{1}[0, \infty)$, then

$$
\lim _{\nu \rightarrow \infty} \int_{0}^{\infty}\left|\sigma_{\nu}(f, x)-f(x)\right| d x=\lim _{\nu \rightarrow \infty} \int_{0}^{\infty}\left|\tilde{\sigma}_{\nu}(f, x)-f(x)\right| d x=0
$$

The next Tauberian type results are interesting in themselves. 
LEMMA 3. If $f \in L^{1}[0, \infty), F$ and $\tilde{F}$ are LAC over $[0, \infty), v>0$, and $\lambda>1$, then

$$
\begin{aligned}
u_{v}(f, x)-\sigma_{v}(f, x)= & \frac{\lambda}{\lambda-1}\left[\sigma_{\lambda v}(f, x)-\sigma_{v}(f, x)\right] \\
& +\tau_{v}(f, \lambda, x)-\frac{1}{(\lambda-1) v} \int_{\nu}^{\lambda v} F(t) \frac{\sin x t}{x} d t, \\
\tilde{u}_{\nu}(f, x)-\tilde{\sigma}_{\nu}(f, x)= & \frac{\lambda}{\lambda-1}\left[\tilde{\sigma}_{\lambda v}(f, x)-\tilde{\sigma}_{v}(f, x)\right] \\
& +\tilde{\tau}_{v}(f, \lambda, x)+\frac{1}{(\lambda-1) v} \int_{\nu}^{\lambda v} \tilde{F}(t) \frac{\cos x t}{x} d t .
\end{aligned}
$$

Proof. By definition,

$$
\begin{aligned}
s_{\nu}(f, x)-\sigma_{\nu}(f, x)= & \int_{0}^{\nu} \frac{t}{\nu} F(t) \cos x t d t, \\
\sigma_{\lambda \nu}(f, x)-\sigma_{\nu}(f, x)= & \frac{\lambda-1}{\lambda} \int_{0}^{\nu} \frac{t}{v} F(t) \cos x t d t \\
& +\int_{\nu}^{\lambda \nu}\left(1-\frac{t}{\lambda \nu}\right) F(t) \cos x t d t .
\end{aligned}
$$

Hence it follows that

$$
\begin{aligned}
s_{\nu}(f, x)-\sigma_{v}(f, x)= & \frac{\lambda}{\lambda-1}\left[\sigma_{\lambda \nu}(f, x)-\sigma_{v}(f, x)\right] \\
& -\frac{\lambda}{\lambda-1} \int_{\nu}^{\lambda v}\left(1-\frac{t}{\lambda \nu}\right) F(t) \cos x t d t .
\end{aligned}
$$

By integration by parts, we get

$$
\begin{aligned}
\int_{v}^{\lambda \nu}\left(1-\frac{t}{\lambda \nu}\right) F(t) \cos x t d t= & -\left(1-\frac{1}{\lambda}\right) F(v) \frac{\sin v x}{x}+\frac{1}{\lambda v} \int_{v}^{\lambda v} F(t) \frac{\sin x t}{x} d t \\
& -\int_{v}^{\lambda v}\left(1-\frac{t}{\lambda v}\right) F^{\prime}(t) \frac{\sin x t}{x} d t .
\end{aligned}
$$

Combining this with (3.3) gives

$$
\begin{aligned}
s_{\nu}(f, x)-\sigma_{\nu}(f, x)= & \frac{\lambda}{\lambda-1}\left[\sigma_{\lambda \nu}(f, x)-\sigma_{\nu}(f, x)\right]+F(\nu) \frac{\sin v x}{x} \\
& -\frac{1}{(\lambda-1) v} \int_{\nu}^{\lambda \nu} F(t) \frac{\sin x t}{x} d t+\tau_{\nu}(f, \lambda, x) .
\end{aligned}
$$

Hence (3.1) follows by (1.5). 
The proof of (3.2) runs along the same lines. We omit it.

The next two Sidon type inequalities are crucial in the proofs of Theorems 1 and 2 below.

LEMMA 4. (See [3].) If $f$ is locally $L^{p}$-integrable over $[0, \infty)$ for some $1<p \leq 2$, then for any $v>0$ we have

$$
\begin{aligned}
& \int_{0}^{\infty}\left|\frac{1}{v} \int_{v}^{2 v} f(t) \frac{\sin x t}{x} d t\right| d x \leq C_{p}\left(\frac{1}{v} \int_{v}^{2 v}|f(t)|^{p} d t\right)^{1 / p}, \\
& \int_{1 / v}^{\infty}\left|\frac{1}{v} \int_{v}^{2 v} f(t) \frac{\cos x t}{x} d t\right| d x \leq C_{p}\left(\frac{1}{v} \int_{v}^{2 v}|f(t)|^{p} d t\right)^{1 / p} .
\end{aligned}
$$

Here and in the sequel, by $C_{p}$ we denote a constant depending only on $p$, whose value may be different at different occurrencies.

\section{Proofs of the results in Section 2}

ProOF OF THEOREM 1. By (3.1), we can estimate as follows

$$
\begin{aligned}
\left|\int_{0}^{\infty}\right| u_{v}(f, x)-\sigma_{\nu}(f, x)\left|d x-\int_{0}^{\infty}\right| \tau_{\nu}(f, \lambda, x)|d x| \\
\leq \frac{\lambda}{\lambda-1} \int_{0}^{\infty}\left|\sigma_{\lambda \nu}(f, x)-\sigma_{\nu}(f, x)\right| d x \\
\quad+\frac{1}{\lambda-1} \int_{0}^{\infty}\left|\frac{1}{v} \int_{v}^{\lambda \nu} F(t) \frac{\sin x t}{x} d t\right| d x=: J_{1}+J_{2},
\end{aligned}
$$

say. By Lemma 2 , for any fixed $\lambda>1$ we have

$$
\lim _{\nu \rightarrow \infty} J_{1}=0 \text {. }
$$

We may assume that $1<\lambda \leq 2$. By (3.4),

$$
J_{2} \leq \frac{C_{p}}{\lambda-1}\left(\frac{1}{v} \int_{\nu}^{\lambda \nu}|F(t)|^{p} d t\right)^{1 / p} .
$$

Making use of Lemma 1 , for any fixed $\lambda>1$ we conclude

$$
\lim _{\nu \rightarrow \infty} J_{2} \leq \lim _{\nu \rightarrow \infty} \frac{C_{p}}{(\lambda-1)^{1 / q}} \sup _{t \geq \nu}|F(t)|=0,
$$

where $1 / p+1 / q=1$. Combining (4.1)-(4.3), we see that

$$
\lim _{\nu \rightarrow \infty} \int_{0}^{\infty}\left|u_{\nu}(f, x)-\sigma_{v}(f, x)\right| d x=0
$$


if and only if condition (2.2) is satisfied.

It remains to take into account that relations (2.1) and (4.4) are equivalent, thanks to Lemma 2. This completes the proof of the equivalence of (2.1) and (2.2).

PROOF OF THEOREM 2. In overall outline, it follows the proof of Theorem 1. This time, we have to use inequality (3.5) instead of (3.4). This is the reason that in (2.3) the integration interval is $[1 / \nu, \infty)$ instead of $[0, \infty)$.

PROOF OF COROLLARY 1. It follows immediately from Theorem 1, Definition (1.5), and the fact that $(\sin v x) / x$ does not belong to $L^{1}[0, \infty)$ for any $v>0$.

PROOF OF COROLlARY 2. It follows immediately from Theorem 2, Definition (1.6), and the fact that $(\cos v x) / x$ does not belong to $L^{1}[1 / v, \infty)$ for any $v>0$.

PROOF OF COROLlaRY 3. By (1.5) and (2.2),

$$
\begin{array}{r}
\left|\int_{0}^{X}\right| s_{\nu}(f, x)-f(x)|d x-| F(v)\left|\int_{0}^{x}\right| \frac{\sin v x}{x}|d x| \\
\leq \int_{0}^{X}\left|u_{\nu}(f, x)-f(x)\right| d x \rightarrow 0 \text { as } \nu \rightarrow \infty,
\end{array}
$$

thanks to Theorem 1. It is easy to check that

$$
C \ln (v X) \leq \int_{1 / \nu}^{X}\left|\frac{\sin v x}{x}\right| d x \leq \int_{0}^{X}\left|\frac{\sin v x}{x}\right| d x \leq 1+\ln (v X) \quad(\nu X \geq 1)
$$

with a constant $C>0$, not depending on $v$ and $X$. From (4.5) and (4.6) we conclude (2.3).

PROOF OF COROLlaRY 4. By (1.6) and (2.4),

$$
\begin{gathered}
\left|\int_{1 / v}^{X}\right| \tilde{s}_{v}(f, x)-f(x)|d x-| \tilde{F}(v)\left|\int_{1 / v}^{X}\right| \frac{\cos v x}{x}|d x| \\
\quad \leq \int_{1 / v}^{X}\left|\tilde{u}_{v}(f, x)-f(x)\right| d x \rightarrow 0 \text { as } v \rightarrow \infty
\end{gathered}
$$

thanks to Theorem 2. Since

$$
C \ln (\nu X) \leq \int_{1 / \nu}^{X}\left|\frac{\cos v x}{x}\right| d x \leq \ln (\nu X) \quad(\nu X \geq 1),
$$


it follows that

$$
\lim _{\nu \rightarrow \infty} \int_{1 / \nu}^{X}\left|\tilde{s}_{\nu}(f, x)-f(x)\right| d x=0 \text { if and only if } \lim _{\nu \rightarrow \infty} \tilde{F}(\nu) \ln \nu=0 .
$$

It remains to take into account that

$$
\lim _{\nu \rightarrow \infty} \int_{0}^{1 / \nu}|f(x)| d x=0
$$

and by definition,

$$
\begin{aligned}
\lim _{\nu \rightarrow \infty} \int_{0}^{1 / \nu}\left|\tilde{s}_{\nu}(f, x)\right| d x & \leq \lim _{\nu \rightarrow \infty} \int_{0}^{1 / \nu} \int_{0}^{\nu}|\tilde{F}(t)| d t d x \\
& =\lim _{\nu \rightarrow \infty} \frac{1}{v} \int_{0}^{\nu}|\tilde{F}(t)| d t=0,
\end{aligned}
$$

due to Lemma 1. This completes the proof of (2.6).

ProOF OF THEOREM 3. By (3.4),

$$
\begin{aligned}
\int_{0}^{\infty}\left|\tau_{\nu}(f, \lambda, x)\right| d x & \leq \frac{\lambda \nu C_{p}}{\lambda-1}\left(\frac{1}{v} \int_{v}^{\lambda \nu}\left(1-\frac{t}{\lambda \nu}\right)^{p}\left|F^{\prime}(t)\right|^{p} d t\right)^{1 / p} \\
& \leq C_{p}\left(v^{p-1} \int_{\nu}^{\lambda \nu}\left|F^{\prime}(t)\right|^{p} d t\right)^{1 / p} \\
& \leq C_{p}\left(\int_{v}^{\lambda \nu} t^{p-1}\left|F^{\prime}(t)\right|^{p} d t\right)^{1 / p}
\end{aligned}
$$

According to Remark (i) made in Section 2 (cf. (2.7')), (2.2) follows.

Proof of Corollary 5. By (2.8), there exists a constant $C$ such that

$$
\int_{v}^{\lambda v} t^{p-1}\left|F^{\prime}(t)\right|^{p} d t \leq C \int_{v}^{\lambda v} \frac{d t}{t}=C \ln \lambda \rightarrow 0 \quad \text { as } \lambda \downarrow 1 .
$$

PROOF OF THEOREM 4. It is similar to the proof of Theorem 3.

PROOF OF COROLlary 6. It is the same as the proof of Corollary 5. 


\section{Extension to complex Fourier transform}

In the sequel, we consider a complex-valued function $g$ that is Lebesgue integrable over $(-\infty, \infty)$, in symbols : $g \in L^{1}(-\infty, \infty)$. The complex Fourier transform of $g$ is defined by

$$
G(t):=\frac{1}{2 \pi} \int_{-\infty}^{\infty} g(x) e^{-i t x} d x \quad(-\infty<x<\infty) .
$$

This time the inversion formula is of the form

$$
g(x)=\lim _{\nu \rightarrow \infty} \int_{-\nu}^{\nu} G(t) e^{i x t} d t
$$

(see for example [5, p. 42]). Accordingly, we introduce the following notations:

$$
\begin{aligned}
s_{v}(g, x) & :=\int_{-v}^{v} G(t) e^{i x t} d t, \\
\sigma_{\nu}(g, x) & :=\int_{-v}^{v}\left(1-\frac{|t|}{v}\right) G(t) e^{i x t} d t, \\
\tau_{v}(g, \lambda, x) & :=\frac{\lambda}{\lambda-1} \int_{\nu \leq|x| \leq \lambda \nu}\left(1-\frac{|t|}{\lambda \nu}\right) G^{\prime}(t) \frac{e^{i x t}}{i x} d t,
\end{aligned}
$$

provided that in the last case $G$ is LAC over $(-\infty, \infty)$, where $v>0, \lambda>1$, and $x \neq 0$ are real numbers. By integration by parts, we obtain

$$
s_{\nu}(g, x)=G(v) \frac{e^{i v x}}{i x}-G(-v) \frac{e^{-i v x}}{i x}-\int_{-v}^{v} G^{\prime}(t) \frac{e^{i x t}}{i x} d t .
$$

This motivates the introduction of the modified partial integral defined by

$$
u_{v}(g, x):=s_{v}(g, x)-G(v) \frac{e^{i v x}}{i x}+G(-v) \frac{e^{-i v x}}{i x} .
$$

Analogously to Theorems 1 and 2, the following is true.

TheOREM 5. If $g \in L^{1}(-\infty, \infty)$ and $G$ is LAC over $(-\infty, \infty)$, then

$$
\lim _{\nu \rightarrow \infty} \int_{|x| \geq 1 / \nu}\left|u_{\nu}(g, x)-g(x)\right| d x=0
$$

if and only if

$$
\lim _{\lambda \downarrow 1} \limsup _{\nu \rightarrow \infty} \int_{|x| \geq 1 / \nu}\left|\tau_{\nu}(g, \lambda, x)\right| d x=0
$$


The counterpart of Corollaries 1 and 2 reads as follows.

COROllary 7. If $g \in L^{1}(-\infty, \infty)$, $G$ is LAC over $(-\infty, \infty)$, and condition (5.3) is satisfied, then $s_{v}(g) \notin L^{1}((-\infty,-1 / \nu) \cup(1 / \nu, \infty))$ for each $v$ such that $|G(v)|+|G(-v)| \neq 0$.

The counterpart of Corollaries 3 and 4 reads as follows.

Corollary 8. If $g \in L^{1}(-\infty, \infty), G$ is LAC over $(-\infty, \infty)$, and condition (5.3) is satisfied, then for any $0<X<\infty$

$$
\lim _{\nu \rightarrow \infty} \int_{-X}^{X}\left|s_{\nu}(g, x)-g(x)\right| d x=0 \quad \text { if and only if } \lim _{|\nu| \rightarrow \infty} G(\nu) \ln |\nu|=0 .
$$

Analogously to Theorems 3 and 4, one can prove the following.

THEOREM 6. If $g \in L^{1}(-\infty, \infty), G$ is LAC over $(-\infty, \infty)$, and for some $p>1$ we have

$$
\lim _{\lambda \downarrow 1} \limsup _{\nu \rightarrow \infty} \int_{\nu \leq|t| \leq \lambda \nu}|t|^{p-1}\left|G^{\prime}(t)\right|^{p} d t<\infty,
$$

then condition (5.3) is satisfied.

CoRollary 9. If $g \in L^{1}(-\infty, \infty), G$ is LAC over $(-\infty, \infty)$, and

$$
t G^{\prime}(t)=\mathscr{O}(1) \text { as }|t| \rightarrow \infty,
$$

then condition (5.5) is satisfied.

Also, conditions (5.5), or in particular (5.6), are sufficient for the fulfillment of both (5.2) and (5.4).

\section{Proofs of the results in Section 5}

First, we formulate the auxiliary results corresponding to Lemmas $1-4$.

Lemma 5. (See [5, p. 1].) If $g \in L^{1}(-\infty, \infty)$, then $G(t)$ is continuous over $(-\infty, \infty)$ and

$$
\lim _{|t| \rightarrow \infty} G(t)=0
$$


LEMMA 6. (See [5, p. 35].) If $g \in L^{1}(-\infty, \infty)$, then

$$
\lim _{v \rightarrow \infty} \int_{-\infty}^{\infty}\left|\sigma_{v}(g, x)-g(x)\right| d x=0 .
$$

LEMMA 7. If $g \in L^{1}(-\infty, \infty), G$ is LAC over $(-\infty, \infty), v>0$, and $\lambda>1$, then

$$
\begin{aligned}
u_{\nu}(g, x)-\sigma_{\nu}(g, x)= & \frac{\lambda}{\lambda-1}\left[\sigma_{\lambda \nu}(g, x)-\sigma_{v}(g, x)\right]+\tau_{v}(g, \lambda, x) \\
& +\frac{i}{(\lambda-1) v}\left[\int_{\nu}^{\lambda v} G(t) \frac{e^{i x t}}{x} d t-\int_{-\lambda \nu}^{-v} G(t) \frac{e^{i x t}}{x} d t\right] .
\end{aligned}
$$

LEMMA 8. (See [3].) If $g$ is locally $L^{p}$-integrable over $(-\infty, \infty)$ for some $1<$ $p \leq 2$, then for any $v>0$ we have

$$
\int_{|x| \geq 1 / v}\left|\frac{1}{v} \int_{\nu}^{\lambda v} g(t) \frac{e^{i x t}}{x} d t\right| d x \leq C_{p}\left(\frac{1}{v} \int_{\nu}^{\lambda \nu}|g(t)|^{p} d t\right)^{1 / p},
$$

and an analogous inequality holds when $\int_{-\lambda \nu}^{-v}$ is substituted for $\int_{v}^{\lambda v}$ on both sides.

Our last lemma is interesting in its own right.

LEMMA 9. If $G$ is $\mathrm{LAC}$ over $(-\infty, \infty)$, then for any $0<X<\infty$,

$$
\lim _{\nu \rightarrow \infty} \int_{1 / \nu \leq|x| \leq X}\left|G(v) \frac{e^{i v x}}{x}-G(-v) \frac{e^{-i v x}}{x}\right| d x=0
$$

if and only if

$$
\lim _{|v| \rightarrow \infty} G(v) \ln |v|=0 .
$$

PROOF. Sufficiency. Relation (6.1) follows immediately from (4.6), (4.7), and (6.2). Necessity. An elementary reasoning and (4.6) yields

$$
\begin{aligned}
I(\nu, X):= & \int_{1 / v \leq|x| \leq X}\left|G(v) \frac{e^{i v x}}{x}-G(-v) \frac{e^{-i v x}}{x}\right| d x \\
= & \int_{1 / v}^{X}\left[\left|G(v) \frac{e^{i v x}}{x}-G(-v) \frac{e^{-i v x}}{x}\right|\right. \\
& \left.\quad+\left|G(v) \frac{e^{-i v x}}{x}-G(-v) \frac{e^{i v x}}{x}\right|\right] d x \\
\geq & |G(v)+G(-v)| \int_{1 / v}^{X}\left|\frac{e^{i v x}-e^{-i v x}}{x}\right| d x \\
\geq & 2 C|G(v)+G(-v)| \ln (\nu X) .
\end{aligned}
$$


Thus, from (6.1) it follows that

$$
\lim _{\nu \rightarrow \infty}|G(\nu)+G(-v)| \ln v=0 .
$$

However, in (6.3) we can estimate in a different way, too. Namely, by (4.7),

$$
\begin{aligned}
I(\nu, X) & \geq|G(\nu)-G(-v)| \int_{1 / \nu}^{X}\left|\frac{e^{i \nu x}+e^{-i \nu x}}{x}\right| d x \\
& \geq 2 C|G(\nu)-G(-v)| \ln (\nu X),
\end{aligned}
$$

whence

$$
\lim _{v \rightarrow \infty}|G(v)-G(-v)| \ln v=0 .
$$

Combining (6.4) and (6.6) gives (6.2).

After these preliminaries, the proofs of Theorems 5, 6 and Corollary 9 follow the same patterns as those of the corresponding results in Section 4 .

Only the proofs of Corollaries 7 and 8 need some explanation.

Proof of Corollary 7. By Theorem $5, u_{v}(g) \in L^{1}(-\infty, \infty)$. Taking into account definition (5.1), it is enough to show that

$$
G(v) \frac{e^{i \nu x}}{x}-G(-v) \frac{e^{-i \nu x}}{x} \notin L^{1}((-\infty,-1 / \nu) \cup(1 / \nu, \infty))
$$

if $v$ is such that at least one of the values $G(v)$ and $G(-v)$ differs from zero. But this is the case since, similarly to (6.3) and (6.5), we have

$$
\begin{aligned}
& I(\nu, \infty) \geq 2|G(v)+G(-v)| \int_{1 / v}^{\infty}\left|\frac{\cos v x}{x}\right| d x, \\
& I(\nu, \infty) \geq 2|G(v)-G(-v)| \int_{1 / v}^{\infty}\left|\frac{\sin v x}{x}\right| d x .
\end{aligned}
$$

Consequently, $s_{v}(g) \notin L^{1}((-\infty,-1 / \nu) \cup(1 / \nu, \infty))$.

PROOF OF COROLlARY 8. By Theorem 5, we have (5.2). Keeping Definition (5.1) in mind, we conclude that, for any $0<X<\infty$,

$$
\lim _{\nu \rightarrow \infty} \int_{1 / v \leq|x| \leq X}\left|s_{\nu}(g, x)-g(x)\right| d x=0
$$

if and only if condition (6.1) is satisfied. By Lemma 9, the latter is equivalent to condition (6.2). 
It remains to take into account that

$$
\lim _{v \rightarrow \infty} \int_{-1 / v}^{1 / v}|g(x)| d x=0
$$

and by Lemma 5 ,

$$
\begin{aligned}
\lim _{\nu \rightarrow \infty} \int_{-1 / \nu}^{1 / v}\left|s_{\nu}(g, x)\right| d x & \leq \lim _{\nu \rightarrow \infty} \int_{-1 / v}^{1 / v} \int_{-\nu}^{\nu}|G(t)| d t d x \\
& =\lim _{\nu \rightarrow \infty} \frac{2}{v} \int_{-\nu}^{\nu}|G(t)| d t=0
\end{aligned}
$$

This completes the proof of (5.4).

\section{References}

[1] W. O. Bray and Č. V. Stanojević, 'Tauberian $L^{1}$-convergence classes of Fourier series II', Math. Ann. 269 (1984), 469-486.

[2] Chang-Pao Chen, ' $L$ '-convergence of Fourier series', J. Austral. Math. Soc. (Series A) 41 (1986), 376-390.

[3] Dăng Vũ Giang and F. Móricz, 'Lebesgue integrability of Fourier transforms', Acta. Sci. Math. (Szeged) 60 (1995), 329-343.

[4] Č. V. Stanojević, 'Structure of Fourier and Fourier-Stieltjes coefficients of series with slowly varying convergence moduli', Bull. Amer. Math. Soc. New Ser. 19 (1988), 283-286.

[5] E. C. Titchmarsh, Introduction to the theory of Fourier integrals (Clarendon Press, Oxford, 1937).

[6] A. Zygmund, Trigonometric series (Cambridge University Press, Cambridge, 1959).

Institute of Mathematics

University of Veszprém

Egyetem u. 10.

8201 Veszprém

Hungary
Bolyai Institute University of Szeged Aradi vértanúk tere 1 6720 Szeged Hungary 